\title{
Indistinguishable of AES-Based PRNG against Modification Attack Based on Statistical Distance Tests and Entropy Measures
}

\author{
Santi Indarjani and Belawati Widjaja
}

\begin{abstract}
Due to previous research, AES-based PRNG is not affected by insertion attack (in random manner) under level of significant $\alpha=0.01$, even it caused some failed tests in randomness. Completing the research, the writers do the observation of the modification attack in random manner against the output sequence of AES-based PRNG that is limited to 1-bit modification attack. The tests are performed by applying statistical distance test between the output sequence before and after the attack. To assure the attack effect, we also measure the entropy values of the sequence before and after attack and compare them. The attack scenario is still the same as the previous research [see 3], except replacing the insertion with modification and parameter $\varepsilon=0.001$. The results show that the modification attack does not give the significant effect on the randomness property of the AES-based PRNG. It was proved from 60 experiments of 1-bit modification attack, that the maximum statistical distances are still far away from $\varepsilon=$ 0.001. And the change of the entropy source after the attack are very small and also still far away from the $\varepsilon=0.001$.
\end{abstract}

Index Terms-AES based PRNG, modification attack, statistical distance, entropy.

\section{INTRODUCTION}

Randomness is a very important role in cryptographic application. The purpose is to guaranty that the critical items such as encryption keys, seed, initialization vector (IV), nonce (number only once), and other parameters that are needed in cryptographic application are random. This lead to assure that the cryptographic system is not weak against the adversary attacks [1].

Some attacks are focus on random generators (RNGs/PRNGs) in order to reduce or even destroy the randomness property of the generators. The attacks can be performed in several approaches, such as compromise the internal state to figure out the seed (input) of the random generator, manipulate the output to make it bias, By destroying the randomness property of the RNG/PRNG, the cryptographic system will fail that lead to the disclosure of the secret value such as key or/and the message. [2]

From previous research with title "Measuring the Insertion attack effect on randomness property of AES-based PRNG", it was proved that the effect of insertion attack is not significant in reducing the randomness property of AES-based PRNG. From total 145 experiments with $10^{3}$

Manuscript received December 24, 2012; revised March 1, 2013.

Santi Indarjani is with National Crypto Institute, Indonesia (e-mail: santi.indarjani@stsn-nci.ac.id, santi_indarjani@yahoo.com).

Belawati Widjaja is with Faculty of Computer Science, University of Indonesia (e-mail : bela@cs.ui.ac.id). samples of sizes $10^{6}$ bits, it was showed that the randomness reduction is about $21,4 \%$ which failed at most only 3 tests in each experiment under level of significantly $\alpha=0.01$. And from the statistical distance test, the output sequences of the AES-based PRNG before and after the insertion attack could not be distinguished under $\varepsilon=0.01$ [3]. Hint previous paper did not include $\mathrm{CBC}$-mode.

In this paper we propose 1-bit modification attack against AES-based PRNG for all variant in each mode of CBC, OFB, CTR and CBC. The purpose is to see the effect of the modification attack against randomness property of AES-based PRNG comparing with the effect of the insertion attack. The scenario attack is still the same with the previous research, only replace the insertion bit process at the pointed location with the bit modification by flipping the target bit into its complement such bit 1 to 0 and vice versa.

From experiment results, it is showed that the modification attack does not give significant effects against the randomness property of the AES-based PRNG. It is proved from the whole statistical distance tests and the entropy measurements that the different between the output sequences before and after the attack is very little and still far away from $\varepsilon=0.001$.

\section{BACKGROUND THEORIES}

\section{A. Advanced Encryption Standard (AES)}

As we know AES is widely used in some security application for encryption and also as PRNG using mode encryption. AES is adopted as a substitution of Data Encryption Standard (DES). AES has 128-bit length of block with three variety of keys are 128-bits, 192-bits and 256-bits. AES parameter can be seen on Table 1 . There are 4 different stages used in a single round of AES i.e. byte substitution, shift row, mix columns, and add round key. For the last round there is no shift row [4].

In a block cipher implementation, there are some modes of encryption can be used to provide the suitable structure in certain application such as develop AES-based PRNG, i.e. Electronic Code Book (EBC), Cipher Block Chaining (CBC), Output Feedback (OFB) and Cipher Feedback (CFB) and Counter Mode (CTR) [4].

\section{B. Concept of Randomness}

All Random sequences generally divided into two classes i.e. truly random sequences and pseudorandom sequences, that produced by RNG and PRNG respectively. RNG is defined as a system whose outputs consists of fully unpredictable (i.e., statistically independent and unbiased) 
bits. In security applications, the unpredictability of the output implies that the generator must be also not observable or even manipulated by any attacker. A true random bit generator usually based on some kind of non-deterministic phenomena. PRNG is defined as a function that, once initialized with some random value (called the seed), outputs a sequences that appears random, in the sense that an observer who does not know the value of the seed cannot distinguish the output from that of a (true) random generator. PRNG is a deterministic process where put it back in the same state will reproduce the same sequence. [5]

\section{Statistical Distance}

Statistical distance is used to see the closeness between two different distributions. Here, an output sequence of AES-based PRNG is assumed as a variable random that has uniform distribution. The distribution is assumed to be changed after the attack. So that to measure whether they are different and can be distinguished, they will be tested using statistical distance.

\section{Definition 1}

Let $x$ and $y$ be random variables taking on values in a finite set $S$. The statistical distance between $x$ and $y$ is defined as [6].

$$
\Delta(x, y)=1 / 2 \sum_{\alpha \in S}|\operatorname{Pr}(x=\alpha)-\operatorname{Pr}(y=\alpha)|
$$

Corollary: If we apply the statistical distance in $\mathrm{n}$ pattern then the statistical distance between $x$ and $y$ become

$$
\Delta(x, y)=\frac{1}{2^{n}} \sum_{\alpha \in S}|\operatorname{Pr}(x=\alpha)-\operatorname{Pr}(y=\alpha)|
$$

\section{Definition 2}

An algorithm $D$ distinguishes $x$ and $y$ with the advantage $\varepsilon$ if and only if

$$
|\operatorname{Pr}(D(x)=1)-\operatorname{Pr}(D(y)=1)| \geq \varepsilon
$$

If the statistical distance between $x$ and $y$ is less than $\varepsilon$ then no algorithm distinguishes $x$ and $y$ with advantage $\varepsilon$. And from other literature it is stated that two random variables are $\varepsilon$-closed if $\Delta(x, y) \leq \varepsilon$ based on the maximum statistical distance [7]

$$
\Delta(x, y)=\max _{\alpha \in S}|\operatorname{Pr}(X n=\alpha)-\operatorname{Pr}(Y n=\alpha)|
$$

Goldreich also said that two ensembles $X$ and $Y$ are statistically close if their statistical difference is negligible, where the statistical difference (also known as variation distance) is defined as the function in (1). He said that if the ensembles $X$ and $Y$ are statistically close, then they are also polynomial-time-indistinguishable [8].

\section{Entropy}

A good pseudorandom generator should be unpredictable. This means that the generation of each output bit should be mutually exclusive and mutually independent one another. Being unpredictable will cause of guessing the next bit is infeasible for an adversary even has some knowledge of previous bits. This property is related to uncertainty of the output sequence, the more uncertain of the sequence the more unpredictable.

\section{Definition 3}

Consider a discreet random variable $X$, with possible outcome $x_{i}, i=1,2, \ldots, \mathrm{n}$. Then the Self information of the event $X=x_{\mathrm{i}}$ is defined as

$$
I\left(x_{i}\right)=\log \left(\frac{1}{P\left(x_{i}\right)}\right)=-\log P\left(x_{i}\right)
$$

Consider a binary source which tosses a fair coin with output a 1 if a head and a 0 if a tail. The information contained of each output is $-\log _{2}(0.5)=1$ bit. Suppose a random sequence $X_{n}=x_{1}, x_{2}, \ldots, x_{n}$ are produced statistically independent by this source, then the total possible outputs are $2^{n}$ with equal probability $1 / 2^{n}$ of each. The information value of an n-bit block is

$$
I\left(x_{i}\right)=-\log _{2} P\left(x_{i}\right)=-\log _{2}\left(2^{-m}\right)=m
$$

Entropy is an uncertainty measurement of a distribution that denotes the average self-information of a random variable.

\section{Definition 4}

Consider a probability distribution $D=p_{1}, p_{2}, \ldots, p_{n}$ then the entropy of $D$ is defined as

$$
H_{b}(D)=-\sum_{i=1}^{n} p_{i} \log _{b} p_{i}=\sum_{i=1}^{n} p_{i} \log _{b} \frac{1}{p_{i}}
$$

\section{E. Attack on PRNG}

The most important problem that can cause the defects on random generator, is a manipulation attempts such as inject a signal to force the output stream bits which is undetected in statistical test. In other words, the random source still passes the randomness statistical tests even it was injected by certain signal. [5].

Goldreich said that modern cryptography is concerned with the construction of schemes that should be able to withstand any abuse, and the schemes are designed so as to maintain a desired functionality, even under malicious attempts aimed at making them deviate from their functionality. An adversary attacking a system will try to manipulate the environment into untypical states. [8].

Other attack is by manipulating a Trojan horse that living in the system to provide the access to get a critical entity produced such as the seed of the random generator or the output stream bits. A Trojan horse also could be manipulated to defect the statistic distribution of output of the random generator such that the output is very sensitive to the input entropy. An attacker also can put a Trojan horse in the RNG/PRNG (hidden) without detectable to perform some attacks such as insertion, deletion or even repetition of the output produced so that the randomness is not a guaranty [10].

\section{EXPERIMENTS AND RESULTS}

\section{A. Experiment Model}

Attack is mounted on each output sequence of all 3 (three) variants of AES with 4 (four) different mode $\mathrm{CBC}$, OFB, 
CFB and CTR. Total random output samples of one variant are 1000 samples with size $10^{6}$ of each sample. The seed that is used to produce the output sequence are the same for all variant and generated using Random C.

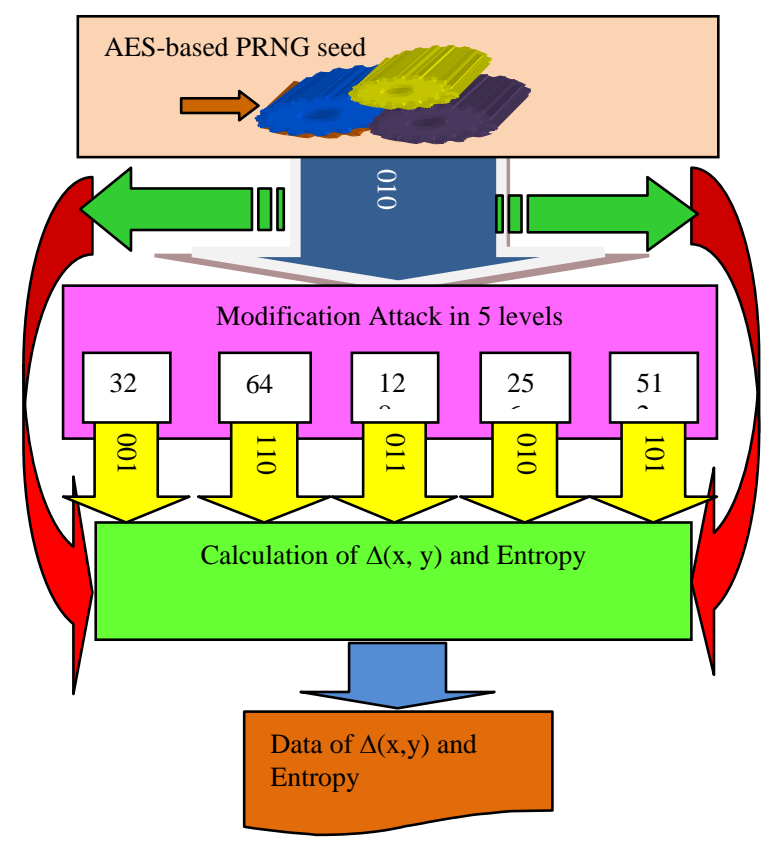

Fig. 1. Experiment model.

Attack is conducted by flipping the pointed bit that is determined based on a random sequence. The location of modification bit is determined based on the decimal value of ${ }^{2} \log \mathrm{b}$ bits where $\mathrm{b}$ bits are taken from a random sequence. The random sequence is also generated by Random $C$. The modification attack is performed in five level blocks are 32-bits, 64-bits, 128-bits, 256-bits and 256-bits. See the experiment model in Fig. 1.

To see the effect of the insertion attack on the randomness property of AES-based PRNG, the sequence before and after modification attack will be evaluated by mounting the statistical distance test. To assure the result of the statistical distance test, the two sequences (before and after the attack) will be evaluated by measuring the entropy value of each and then compare them to see any change of the entropy value after the attack.

First, we assume that the two output sequences (from the AES-based PRNG) before and after the attack are having different distribution because of the attack. Our hypothesis that the two sequences can be distinguished under a little parameter of $\varepsilon=0.001$ (different with previous research that used strengthen parameter, i.e., $\varepsilon=0.01$ ).

Here we measure the statistical distance based on the maximum distance and the values of $\Delta(x, y)$. If the maximum statistical distance between two distribution is less then $\varepsilon=0.001$ then the two distributions are $\varepsilon$-close or the two distribution could not be distinguished. This meant that the modification attack does not give the effect significantly on the randomness property of the PRNG. On the other side, if the maximum statistical distance are bigger than the $\varepsilon=0.001$ then we could say the two sequences can be distinguished under the advantage of $\varepsilon=0.001$. This leads to the conclusion that the attack has the significant attack on the PRNG.
To assure the results, we also measure the entropy value of the sequence before and after the attack. And we see whether the difference is significant or not by using the same parameter $\varepsilon=0.001$. If the difference bigger than $\varepsilon=0.001$ than we could say that the attack has the significant effect on the randomness of the PRNG, otherwise not.

The tests are mounted based on non overlapping pattern 1 , 2, 3, 4 and 8 to see the effect changes on the PRNG.

\section{B. Results}

The experiment results show that the modification attack does not affect significantly on the AES-based PRNG. The maximum statistical distance for AES-128, AES-192 and AES-256 are 0.00002885, 0.0000237 and 0.00002578 respectively. All the values occurred on CBC-mode in 32-bit level, which are still far away from the parameter $\varepsilon=0.001$.

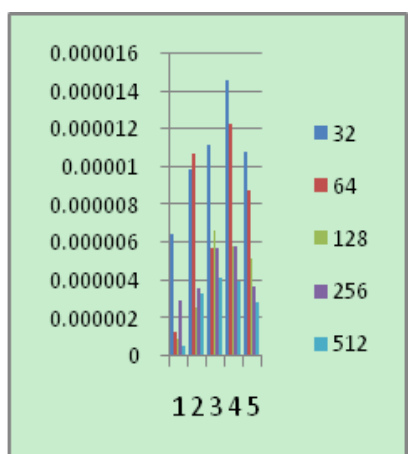

a). Max statistical distance

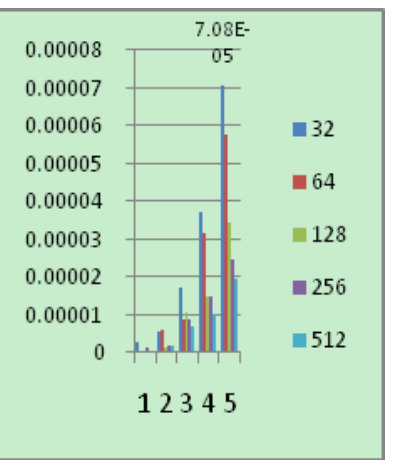

b). Max entropy difference
Fig. 2. Modification attack effect on AES-128 CTR.

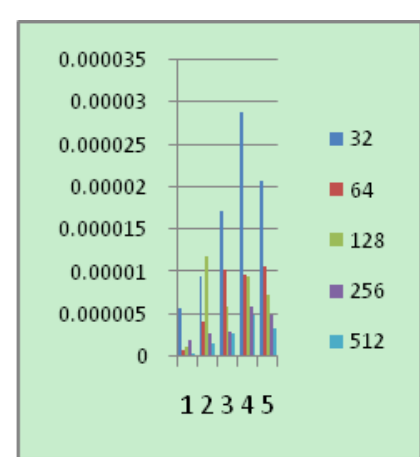

a). Max statistical distance

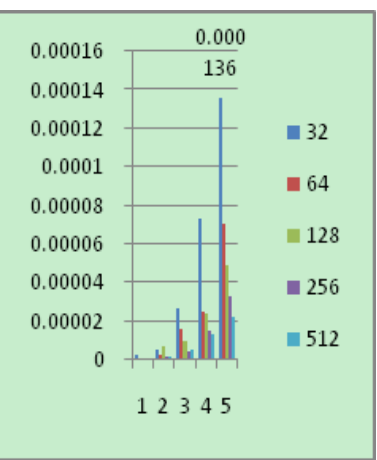

b). Max entropy difference
Fig. 3. 1-bit modification attack effect on AES-128 CBC.

The example of experiment results of the attack on AES-128 with mode CTR, CBC, CFB and OFB are presented in Fig. 2 to Fig. 5, respectively. First part of each figure shows all maximum statistical distance values from each experiment (part a) and second part shows all maximum entropy difference values obtained from each experiment (part b). On part b, we specify the highest maximum entropy value.

From the results, we could see that in general the attack gives more effect on level block 32-bit of modification and will decrease for the bigger size (64-bit, 128-bit, 256-bit and 512-bit). It happened because bigger size of block caused lesser bit to be changed.

Because all the maximum distance values resulted still far away under $\varepsilon=0.001$, then to see the attack effect on each variance or mode, then we focus on statistical distance values that are bigger than 0.00001 . 


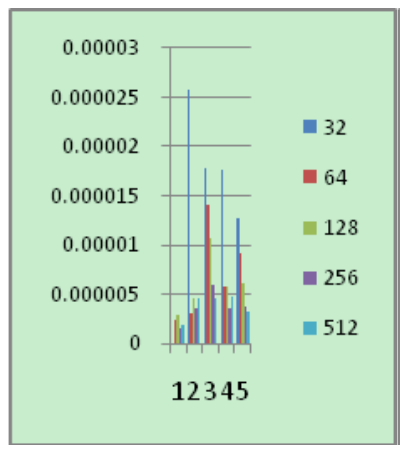

a). Max statistical distance

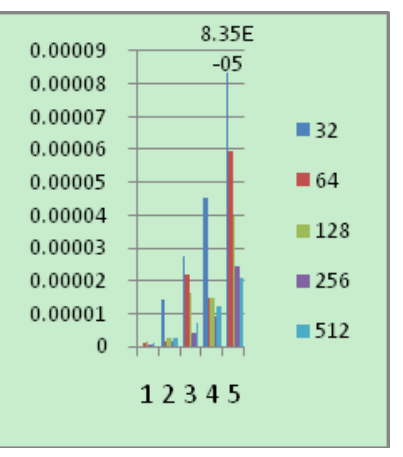

b). Max entropy difference
Fig. 4. 1-bit modification attack effect on AES-128 CFB.

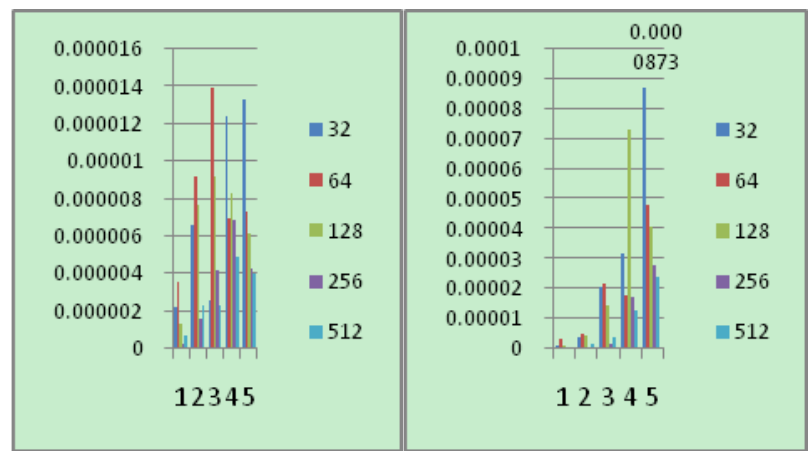

a). Max statistical distance

b). Max entropy difference

Fig. 5. 1-bit modification attack effect on AES-128 OFB.

From all variances, we identify that total maximum statistical distance that bigger than 0.00001 for all modes are close, but we can see that mode CTR has the lowest amount which indicates that the attack effect on this mode is less significant than others (see Table I). On the other side, mode CBC got the worse effect. And from the entropy measurements, as we can see on Table II, the maximum entropy difference values for each mode on each variance are still less than 0.0001 .

TABLE I: COMPOSITION OF MAX STATISTICAL DisTANCE VALUE $>0.00001$ ON EACH VARIANCE

\begin{tabular}{|l|l|l|l|}
\hline & AES-128 & AES-192 & AES-256 \\
\hline CTR & $5(20 \%)$ & $3(15 \%)$ & $3(15 \%)$ \\
\hline CBC & $6(24 \%)$ & $6(24 \%)$ & $9(36 \%)$ \\
\hline CFB & $6(24 \%)$ & $5(20 \%)$ & $6(24 \%)$ \\
\hline OFB & $3(12 \%)$ & $5(20 \%)$ & $6(24 \%)$ \\
\hline
\end{tabular}

TABLE II: MAXIMUM DIFFERENCE ENTROPY VALUES ON EACH MODE

\begin{tabular}{|l|c|l|l|}
\hline & AES-128 & AES-192 & AES-256 \\
\hline CTR & 0.00007079 & 0.00008018 & 0.00007546 \\
\hline CBC & 0.00013602 & 0.00015587 & 0.00016850 \\
\hline CFB & 0.00008354 & 0.00007970 & 0.00007328 \\
\hline OFB & 0.00008734 & 0.00008229 & 0.00008171 \\
\hline
\end{tabular}

These all biggest entropy difference values occurred in level block 32-bit on pattern 8 . The interesting facts that all the biggest maximum statistical distance value on each variance, occurred on mode CBC on level block 32-bit. On Table II, we can also see that the entropy difference on mode $\mathrm{CBC}$ is bigger than the other modes. This indicates that the modification attack affects AES-based PRNG with mode BC more significantly than other modes.

Furthermore, if it will be analyzed more whether each of
AES-variance is differently affected against the modification attack based on randomness test using NIST tools. As a slight comparison from previous research, the mode CTR has the worst effect against the insertion attack, otherwise the mode CFB is less affected against the insertion attack. This contradictive with the fact in the modification attack results where is mode CTR in this case got the less effect.

From a little comparison between the result of 1-bit insertion attack on AES-128 mode OFB (see Fig. 4) and on AES-192 mode OFB (see Fig. 6), we can find an interesting fact where the total values that achieve 0.00001 or more on Fig. 6 is 20 values or $80 \%$ that very further than occurred on Fig. 4 which only has 6 values or $24 \%$.

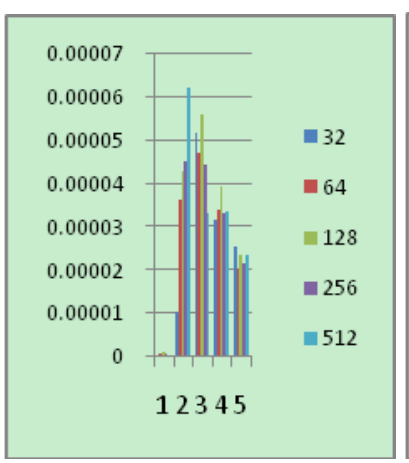

a). Max statistical distance

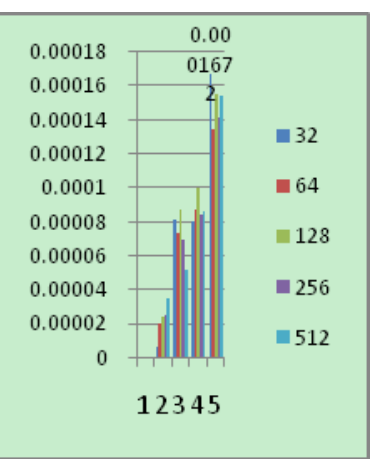

b). Max entropy difference ect on AES-192 CFB.
TABLE III: THE COMPARISON 1-BIT MODIFICATION ATTACK AND 1-BIT INSERTION ATTACK AES-128

\begin{tabular}{|c|c|c|c|c|c|c|}
\hline & \multicolumn{7}{|c|}{ 1-bit random insertion } \\
\hline & max stat distance difference & \multicolumn{2}{c|}{ max entropy difference } \\
\hline & 32 & 64 & 128 & 32 & 64 & 128 \\
\hline OFB & 0.0000400 & 0.0000431 & 0.0000416 & 0.0001255 & 0.0001364 & 0.0001453 \\
\hline CTR & 0.0000397 & 0.0000373 & 0.0000368 & 0.0001194 & 0.0001194 & 0.0001340 \\
\hline CFB & 0.0000518 & 0.0000472 & 0.0000559 & 0.0001672 & 0.0001343 & 0.0001556 \\
\hline & \multicolumn{7}{|c|}{1 -bit modification } & & \\
\hline & max stat distance difference & \multicolumn{2}{|c|}{ max entropy difference } \\
\hline & 32 & 64 & 128 & 32 & 64 & 128 \\
\hline OFB & 0.0000206 & 0.0000103 & 0.0000104 & 0.0000873 & 0.0000479 & 0.0000732 \\
\hline CTR & 0.0000225 & 0.0000098 & 0.0000075 & 0.0000755 & 0.0000570 & 0.0000489 \\
\hline CFB & 0.0000179 & 0.0000125 & 0.0000094 & 0.0000111 & 0.0000068 & 0.0000044 \\
\hline
\end{tabular}

This indicates that on this case, the 1-bit insertion attack gives more significant effect rather than the modification attack. It might be because the insertion attack changes not only the pattern but also cause the position shifting of the consecutive bits at some places. Otherwise the modification attack only changes the pattern but not cause the position change or shifting bits.

To see more deeply we provide the comparison of the two attacks on AES-128 with mode OFB, CTR and CFB in block level 32-bit, 64-bit and 128-bit (see Table III). We can see that all the values on the insertion attack are bigger than on the modification attack. This indicates that the last attack give less effect than the first. To get more complete results, we will explore more this comparison (in progress).

From the measurement of the entropy value we could see that the difference between the sequence before and after the attack still very small and far away from the parameter $\varepsilon=$ 0.001. Even the biggest statistical distance value for each variance are still less than 0.0001 which is indicates that the 
1-bit modification attack does not give the significant effect under $\varepsilon=0.001$.

From the results we could said that there is no significant change on the entropy value of each two compared sequences. This means that the entropy after the attack is still close to the entropy value before the attack. It leads to the thought that the modification attack doesn't give the significant effect against the AES-based PRNG. This strengthened the statistical distance test results.

So from the two measurements that provide all values still smaller then parameter $\varepsilon=0.001$, then we come to the conclusion that the output sequences from the AES-based PRNG before and after the attack still could not be distinguished under $\varepsilon=0.001$.

And from the partial comparison between the 1-bit modification attack and the 1-bit insertion attack, we see that the first attack has the smaller value that indicates the modification attack has less significant effect than the insertion attack.

\section{CONClusion AND OPEn PROBlem}

Based on the experiments, we conclude that the modification attack does not affect significantly against the randomness property of the AES-based PRNG under advantage of $\varepsilon=0.001$. And the modification attack also does not cause the significant change on the entropy values of the output of the AES-based PRNG under advantage of $\varepsilon=0.001$.

To completing the research, it is still need to extend the research on other PRNG algorithm or stream cipher to see whether this attack does not give the effect significantly only on AES or also on the other algorithm. And also it is still need to explore the theoretical approach to prove why this modification attack does not affect the randomness property of the output sequences of an AES-based PRNG.

\section{ACKNOWLEDGMENT}

Thanks to Dr. Kiki from Mathematical Department,
University of Indonesia and Dr. Ade from Computer Science faculty, University of Indonesia for the idea to extend the research into modification attack. Thanks to our friends Adi Nugraha, Kempo and Gigih from National Crypto Institute for help us in performing the experiments

\section{REFERENCES}

[1] D. Eastlike, J. Schiller, and S. Crocker. (2005). Randomness Requirements for Security, RFC 4086 (obsoletes RFC1750). [Online] Available: http://www.ietf.org/rfc/rfc4086.txt

[2] N. Ferguson and B. Schneier, Practical Cryptography, Wiley Publishing, Inc., Indianapolis, USA, 2003.

[3] S. Indarjani and B. Widjaja, "Measuring the insertion attack effect on randomness property of AES-based PRNG," IPCSIT, vol. 40 2012, pp. 118-122.

[4] S. William, Cryptography and Network Security: Principles and Practices, $4^{\text {th }}$ ed., Pearson Education, Inc., 2005.

[5] H. C. A. V. Tilborg, Encyclopedia of Cryptography and Security, Springer, USA, 2005.

[6] Farashahi, Schoemaker, and Sidorenko, "Efficient of Pseudorandom Generators based on DDH Assumption," in Proc. the $10^{\text {th }}$ PKC conference, Spriinger-verlag, Berlin, 2007.

[7] Y. Wang, "A comparison of two approaches to the randomness," Theor. Comput. Sci., vol. 276, no. 1-2, pp. 449-459, 2002.

[8] O. Goldreich, Foundation of Cryptography: Volume I Basic Tools, England: Cambridge University Press, 2001.

[9] R. Bose, Information Theory, Coding and Cryptography, Tata McGraw Hill, New Delhi, 2002.

[10] Y. Adam and Y. Moti, Malicious Cryptography, USA: John Willey \& Sons, 2004,

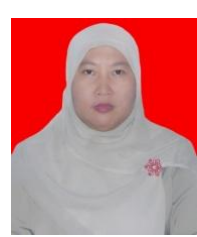

Santi Indarjani was born in Jakarta, Indonesia, on August 29, 1969. She is in Academic Staff of National Crypto Institute, Indonesia. She is in Magister of Information System Management, University of Gunadarma, Indonesia in 2006. She roles as a student of Doctoral Program in Faculty of Computer Science, University of Indonesia since September 2010.

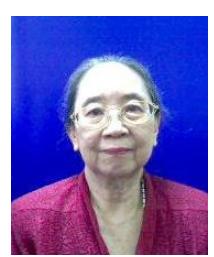

Belawati Widjaja is in Academic Staff of Faculty of Computer Science, University of Indonesia. She obtained her Doctor of Philosophy, Department of Mathematics, University of Kentucky, USA in 1968. 